\title{
A case of massive aortic thrombosis with catastrophic cerebrovascular embolization and infarction
}

\author{
Aleksandra Mamorska-Dyga, Doru Paul, Ch. Khalil, \\ Ghulam Sajjad Khan
}

\section{ABSTRACT}

Introduction: Antiphospholipid syndrome (APS) is the most common type of acquired thrombophilia. Lupus anticoagulant (LA) is more commonly associated with venous than with arterial thrombosis and accounts for $6-8 \%$ of thrombotic events. Case Report: A 50-yearold African-American male presented to the emergency department with right sided hemiparesis, facial droop and impaired speech. First symptoms were noted about 10-12 hours before presentation to the emergency department. During two weeks preceding admission, patient experienced several transient episodes of similar neurological deficits. Computed tomography (CT) scan of brain done on admission showed ischemic stroke involving the area of distribution of the left middle cerebral artery. The CT angiography of brain revealed a large thrombus in the aortic arch, left common carotid and internal carotid artery. Laboratory studies were positive for lupus anticoagulant. Serial brain CT scan demonstrated increasing brain edema and midline shift eventually leading to transtentorial herniation. Several days after admission, neurological examination revealed loss of brain stem reflexes. Conclusion: Antiphosphotipid syndrome (APS) is the most common type of acquired thrombophilia. Lupus anticoagulant is

Aleksandra Mamorska-Dyga1, Doru Paul ${ }^{1}$, Ch. Khalil ${ }^{1}$, Ghulam Sajjad Khan ${ }^{1}$

Affiliations: ${ }^{1}$ Lincoln Medical and Mental Health center, Department of Hematology Oncology

Corresponding Author: Aleksandra Mamorska-Dyga 234 East 149th, Bronx, New York, USA, 10451; Ph: 718579 4937; Fax: 718579 5722; Email: amdyga@gmail.com

Received: 15 March 2012

Accepted: 04 June 2012

Published: 01 June 2013 mainly associated with venous thrombosis and accounts for $6-8 \%$ of thrombotic events in otherwise healthy patients. Prompt diagnosis and treatment, with long-term anticoagulation for survivors is crucial. The most common site of arterial thrombosis in APS is central nervous system, presenting with stroke and transient ischemic attacks in $50 \%$ of the cases. Patients with diagnosed APS and prior thrombosis have high risk for recurrent events. Due to this fact some authors postulate lifelong anticoagulation therapy.

Keywords: Antiphospholipid thrombosis syndrome, Acquired thrombophilia, Aortic thrombosis, Systemic embolization, Cerebrovascular embolization

$* * * * * * * * *$

Mamorska-Dyga A, Paul D, Khalil C, Khan GS. A case of massive aortic thrombosis with catastrophic cerebrovascular embolization and infarction. International Journal of Case Reports and Images 2013;4(6):294-298.

$$
* * * * * * * *
$$

doi:10.5348/ijcri-2013-06-316-CR-1

\section{INTRODUCTION}

Stroke is one of the leading causes of morbidity and mortality in the United States, with an incidence of over 790,000 per year. Eighty seven percent of strokes are ischemic and 25\% affect patients younger than 65 years of age. Before the age of 65 years more women than men suffer from stroke, however the opposite is true for age 65 years and older, as reported by American Heart and American Stroke Association. 
Antiphospholipid syndrome (APS) is the most common type of acquired thrombophilia, associated with venous and/or arterial thrombosis. Left atrial is responsible for $6-8 \%$ of thrombotic events affecting otherwise healthy individuals and is most commonly associated with venous thrombosis $[1,2]$.

We present a case of massive aortic thrombosis with subsequent catastrophic cerebrovascular embolization and infarction.

\section{CASE REPORT}

A 50-year-old African-American male presented with right sided weakness, facial droop and impaired speech. Symptoms started about 10-12 hours before presentation to the emergency department and were gradually getting worse. During two weeks preceding admission patient experienced several episodes of similar symptoms, but less severe, resolving spontaneously, for which he did not seek medical help.

Past medical history was significant for hypertension and stroke two years before, which resolved with no residual deficits. Social history revealed active use of tobacco, alcohol and cocaine.

On admission, patient had markedly elevated blood pressure $(189 / 120 \mathrm{mmHg})$, was tachycardic $(120 / \mathrm{min})$ and had increased respiratory rate $(20 / \mathrm{min})$. Neurological examination revealed equal and reactive to light pupils with gaze deviated to the left, global aphasia, right sided facial droop and right sided hemiparesis.

Computed tomography (CT) scan of brain revealed increased density of the left middle cerebral artery (MCA) and ischemic stroke involving the area of its distribution (Figures 1 and 2). The angiography showed a large thrombus involving the aorta (Figures 3-5), extending to the left common and internal carotid artery and proximal left cerebral artery segments.

Initial management did not include systemic thrombolysis due to prolonged time relapsed since the symptoms were first noted. Patient was also not a candidate for local intervention due to extensiveness of the thrombus.

Patient was intubated for airway protection and admitted to the medical intensive care unit for further management. Treatment was supportive. His hospital course was complicated by aspiration pneumonia, sepsis, acute kidney injury, hemolytic anemia and thrombocytopenia. Laboratory tests were scheduled and revealed increased D-dimers and low haptoglobin levels (Table 1). Peripheral smear was remarkable for the presence of schistocytes, which was suggestive of disseminated intravascular coagulation, which was attributed to sepsis. In view of a possible underlying autoimmune pathology additional blood tests were added, among them mixing studies, which were positive for lupus anticoagulant.

Transthoracic echocardiography was ordered and did not reveal any underlying structural cardiovascular pathology.

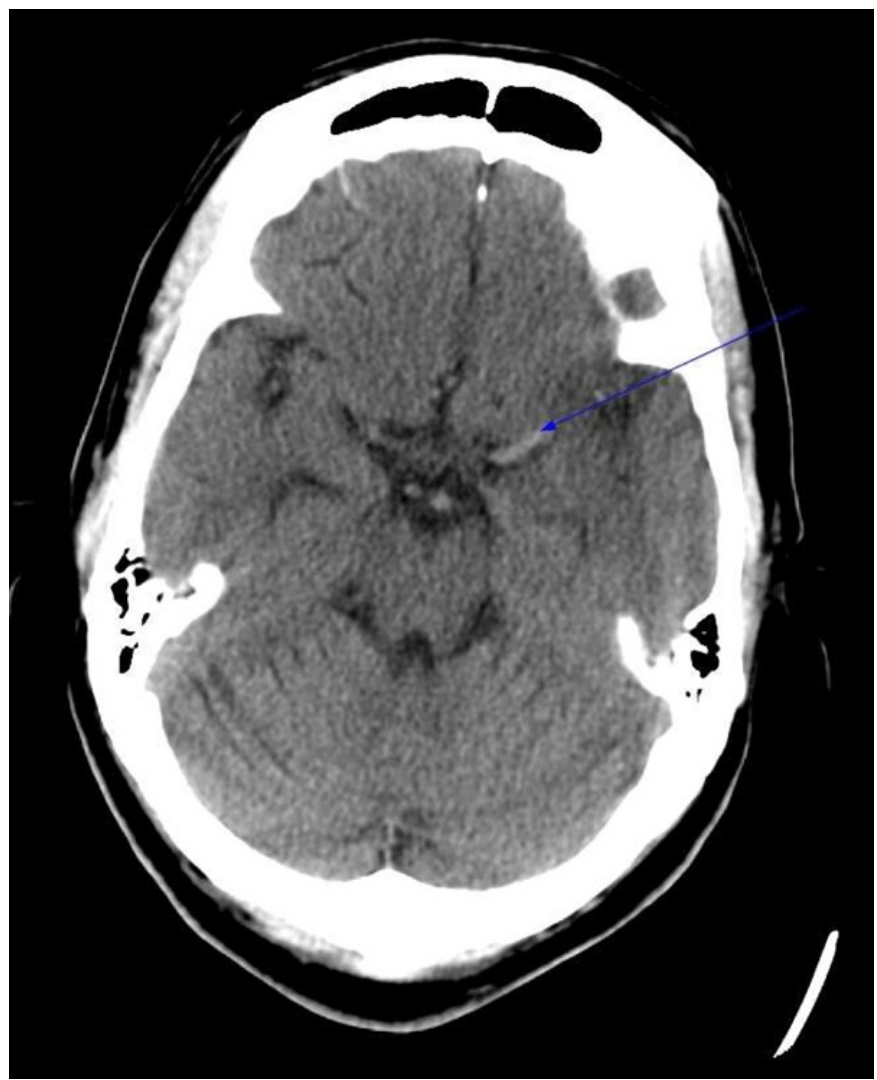

Figure 1: Brain computed tomography showing increased density of the left MCA, marked with an arrow.

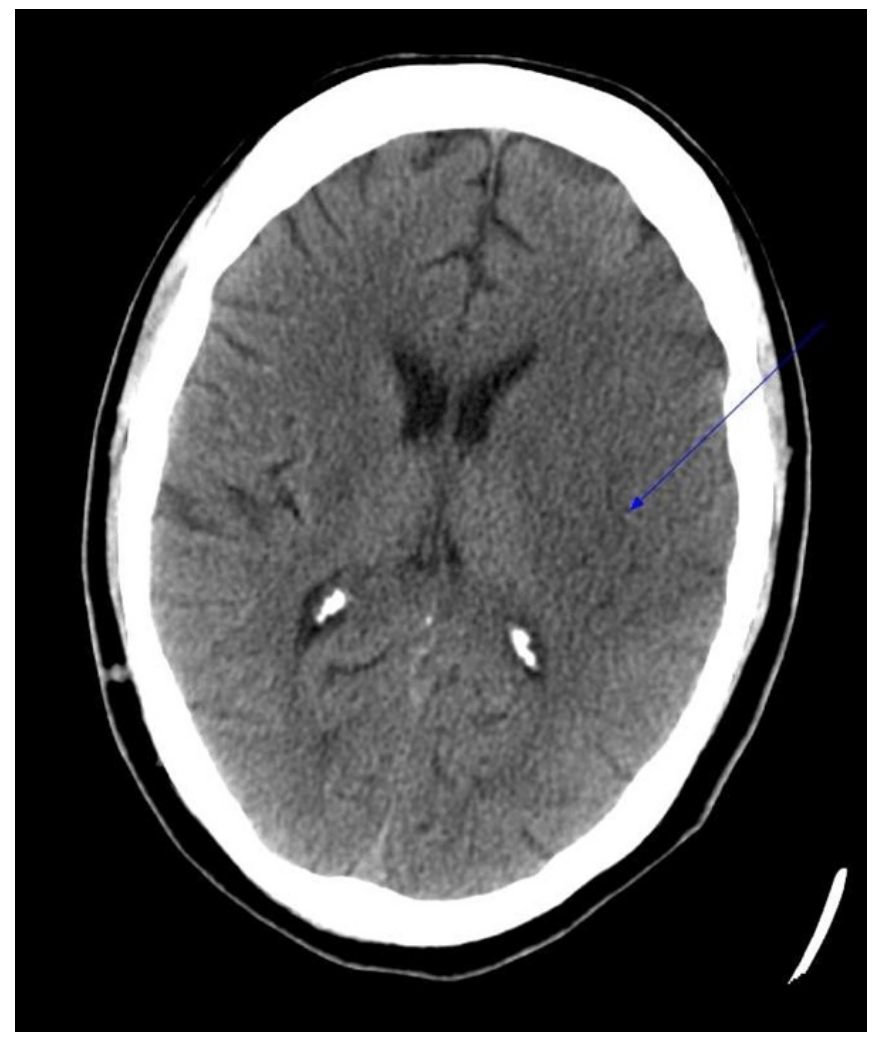

Figure 2: Brain computed tomography showing effacement of the sulci and decreased attenuation in the left parietal lobe, consistent with an early left MCA infarct (arrow pointing to that area). 


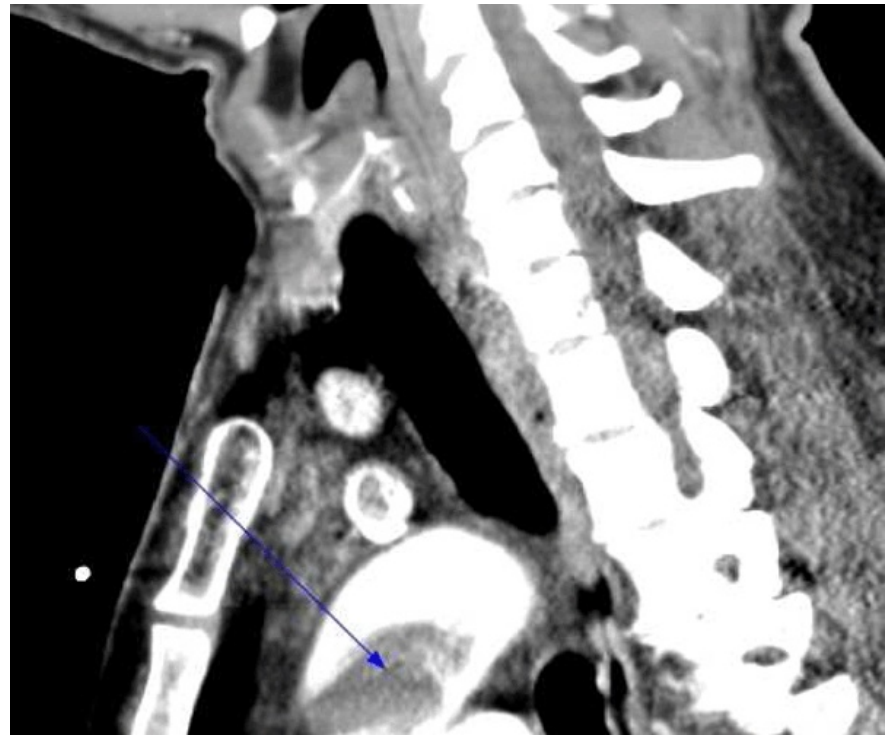

Figure 3: Computed tomography angiogram showing a large thrombus in aortic arch marked with an arrow.

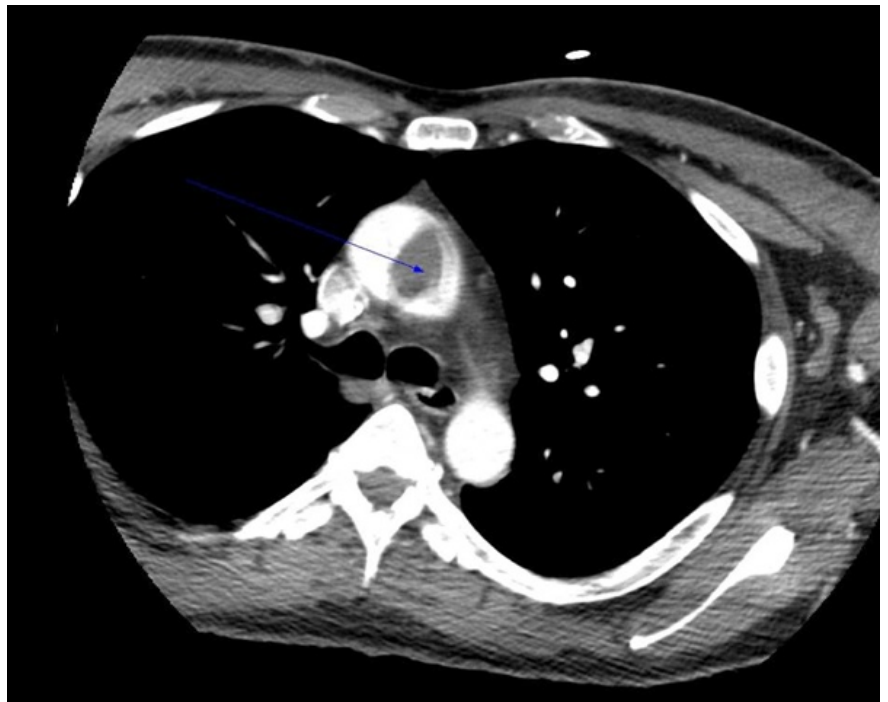

Figure 4: Computed tomography angiogram showing thrombosis in the ascending aorta marked with an arrow.

Serial CT scans showed worsening demarcation of the infarct in the left brain hemisphere, initially with mild mass effect. After several days of hospitalization neurological examination revealed absence of brain stem reflexes. The CT scan of brain obtained at that time showed massive brain edema, midline shift, transtentorial herniation and new brain stem infarct (Figure 6).

\section{DISCUSSION}

Antiphospholipid syndrome, is the most common type of acquired thrombophilia. Within the spectrum of the APS, anticardiolipin antibody syndrome is most commonly associated with arterial thrombosis, as opposed to left atrial, which is mainly related with venous thrombosis [3].

According to the International Consensus Statement on Preliminary Criteria for the classification of APS (Sapporo Criteria), at least one clinical and at least one

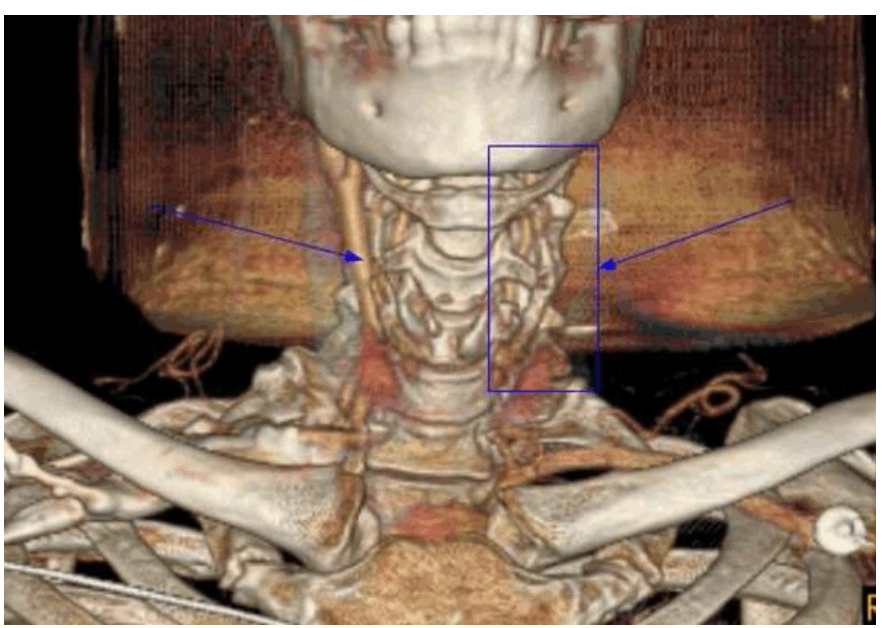

Figure 5: Helical computed tomography angiogram showing filling defect in the left common carotid artery resulting in its complete occlusion. Right common carotid artery marked with an arrow. Left common carotid artery is not visualized (defect marked with box and arrow).

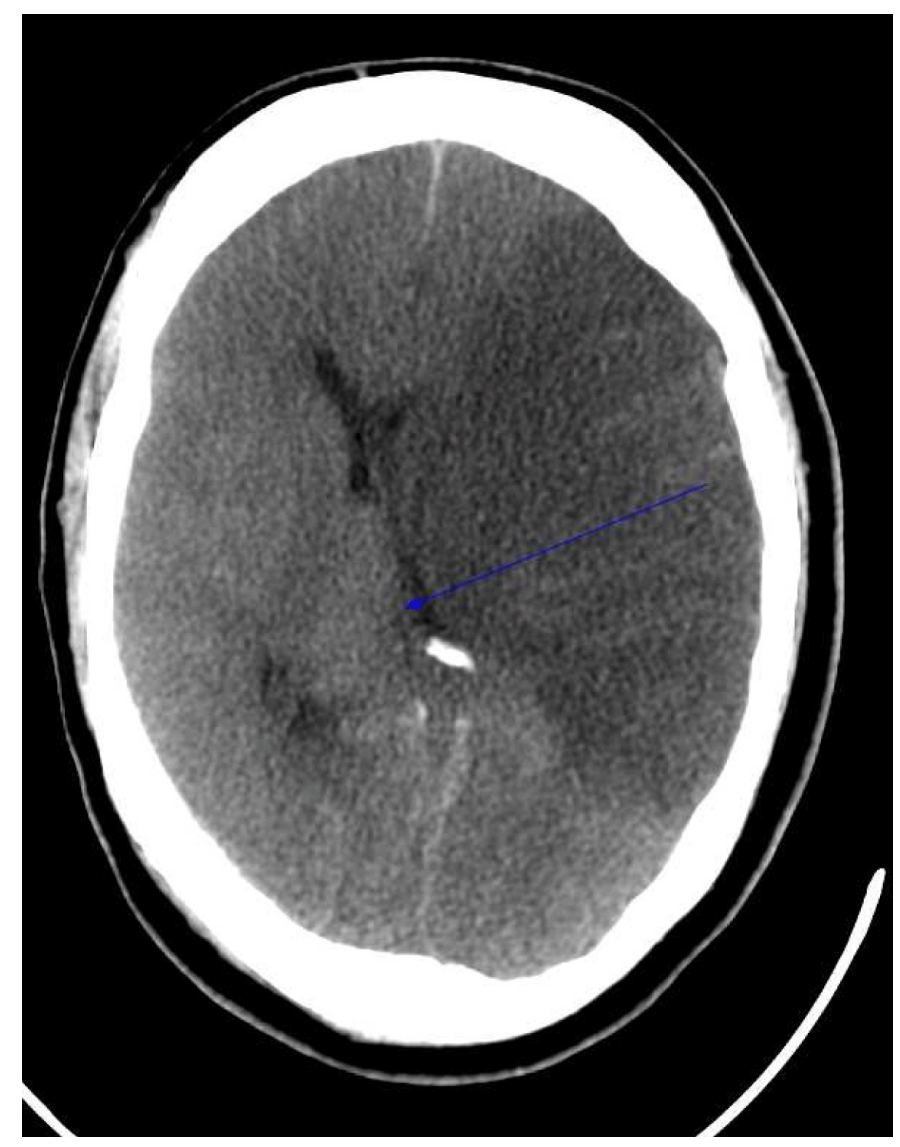

Figure 6: Brain computed tomography showing acute brainstem infarction evolving acute infarction in the left middle cerebral artery distribution. Interval progression of brain edema and mass effect, subfalcine herniation and midline shift to the left (arrow). 
Table 1: Laboratory results of the patient.

\begin{tabular}{lll}
\hline Test & Results & Normal \\
\hline Hgb & $13.4-9.2$ & $13.5-17 \cdot 5 \mathrm{mg} / \mathrm{dL}$ \\
PLT & $243-91$ & $150-350 \times 10^{5} / \mathrm{mm}^{5}$ \\
TP/aPTT & $11.4 / 35.8$ & $11-13 / 25-35 \mathrm{sec}$ \\
HPF-4, SRA & Negative & Negative \\
LDH & 1839 & $11-190 \mathrm{U} / \mathrm{L}$ \\
Haptoglobin & $<10-50$ & $30-200 \mathrm{mg} / \mathrm{dL}$ \\
D-dimers & $>5000$ & $<500 \mathrm{mg} / \mathrm{dL}$ \\
Fibrinogen activity & 557 & $150-400 \mathrm{mg} / \mathrm{dL}$ \\
CD 55, CD 59 & Negative & Negative \\
G-6-PD & $5 \cdot 9$ & $5-13 \mathrm{U} / \mathrm{g} \mathrm{Hb}$ \\
RF & Negative & Negative \\
ANA titer & Positive, speckled & $<1: 160$ \\
& pattern & \\
APL panel & + inhibitor in & \\
& mixing studies & \\
\hline
\end{tabular}

\section{ABBREVIATIONS}

Hb; Hemoglobin, PLT; Platelet, PT; Prothrombin time, aPTT; Activated Partial 120 Thromboplastin Time, INR; International Normalized Ratio, HPF-4, SRA; Heparin Platelet Factor 4, Serotonin Release Assay, LDH; Lactate Dehydrogenase, Fibgn; Fibrinogen, G-6-PD; Glucose 6 phosphate dehydrogenase, RF; Rheumatoid factor, ANA; Antinuclear Antibody, APL; Antiphospholipid.

laboratory criteria are required to consider the APS diagnosis definite. The laboratory criterion is met when the positive test is reproduced in at least 12 weeks interval. Our patient presented with unexplained thromboembolic event and laboratory studies revealed the presence of left atrial. However, the latter could not be repeated due to the fatal outcome.

The most common site of arterial thrombosis in APS is central nervous system, with half of the cases resulting in strokes and transient ischemic attacks (TIAs) [3-5]. The major source of arterial systemic emboli is the heart, whereas systemic thrombi originating in the aorta are much less common. The occurrence of the thrombi without underlying predisposing conditions such as structural heart disease or atherosclerotic plaque would make the case even more unusual [6].

In our patient, no underlying pathology was found in transthoracic echocardiography. While transesophageal study would be a better modality in search for the pathology, in view of his critical condition this test was not performed in our patient.

According to reviewed literature [1], the presence of coexisting mixed aortic, carotid and cerebrovascular thrombosis and/or TIAs (reported in our case prior to admission) is very uncommon among patients with APS, contributing to less than $10 \%$ cases.

The majority of patients developing APS is otherwise healthy, and is classified to have primary APS. Secondary APS should also be considered, especially in the context of medication/drug induced scenario, including cocaine use.
Patients with the diagnosis of APS and prior thrombosis are at high risk for recurrent events. Some authors report, that about $70 \%$ of them will have an episode in 5-6 years, with highest rate during the first six months after discontinuation of the therapy $[4,7,8]$. Due to that fact lifelong anticoagulation therapy is postulated.

Thrombosis occurring at the level of capillaries, arterioles or venules may result in a clinical picture similar to hemolytic uremic syndrome or thrombotic thrombocytopenic purpura [3, 7] which could explain some of the clinical and laboratory findings in our patient.

Initial management did not include systemic thrombolysis as eligible patients are those presenting within three hours of onset of symptoms [9].

\section{CONCLUSION}

Antiphospholipid syndrome is the most common type of acquired thrombophilia. Secondary Antiphospholipid syndrome should be considered in view of the history of recent cocaine use as a provoking factor. Left atrial is mainly associated with venous thrombosis. Concomitant arterial, mixed aortic, carotid and cerebrovascular thrombosis represent a very rare phenomenon, which makes presented case unique.

The prompt diagnosis and treatment, with long-term anticoagulation given to survivors is crucial, as the rate of recurrent episodes is high and the outcomes may be fatal.

$* * * * * * * * *$

\section{Author Contributions}

Aleksandra Mamorska-Dyga - Conception and design, Analysis and interpretation of data, Drafting the article, Critical revision of the article, Final approval of the version to be published

Doru Paul - Conception and design, Acquisition of data, Analysis and interpretation of data, Drafting the article, Critical revision of the article, Final approval of the version to be published

Ch, Khalil - Conception and design, Acquisition of data, Analysis and interpretation of data, Critical revision of the article, Final approval of the version to be published Ghulam Sajjad Khan - Acquisition of data, Analysis and interpretation of data, Critical revision of the article, Final approval of the version to be published

\section{Guarantor}

The corresponding author is the guarantor of submission.

\section{Conflict of Interest}

Authors declare no conflict of interest.

\section{Copyright}

(C) Aleksandra Mamorska-Dyga et al. 2013; This article is distributed under the terms of Creative Commons 
Attribution 3.0 License which permits unrestricted use, distribution and reproduction in any means provided the original authors and original publisher are properly credited. (Please see www.ijcasereportsandimages.com /copyright-policy.php for more information.)

\section{REFERENCES}

1. Rodger L Bick. Antiphospholipid Thrombosis Syndromes. Clin Appl Thrombosis Hemostasis 2001;7(4):241-58.

2. Monica Galli. Antiphospholipid antibodies and thrombosis: do test patients identify the patient's risk? Thrombosis Research 2004;114(5-6):597-601.

3. Bick RL, Baker WF. Antiphospholipid syndrome and thrombosis. Semin Thromb Hemost 1999;25(3);333-50.

4. Ware Branch D, Eller AG. Antiphospholipid Syndrome and Thrombosis. Clinical Obstetrics and Gynecology 2006;49(4):861-74.

5. Rosove MH, Brewer PM. Antiphospholipid thrombosis: clinical course after first thrombotic event in 70 patients. Ann Intern Med 1992;117(4):303-8.

6. Song IW, Hong GR, Cho JH, et al. A case of huge thrombus in the aortic arch with the cerebrovascular embolization. J Cardiovascular Ultrasound 2009;17(4):148-50.

7. Khamashta MA, Cuadraro MJ, Mujic F, Taub NA, Hunnt BJ, Hughes GR. The management of antiphospholipid-antibody syndrome. N Eng J Med 1995;332(15);993-7.

8. Levine JS, Branch DW, Rauch J. The Antiphospholipid Syndrome. N Engl J Med 2002;346(10):752-63.

9. Panetta T, Thompson JE, Talkington CM, Garrett WV, Smith BL. Arterial embolectomy: a 34-year experience with 400 cases. Surg Clin North Am 1986;66(2):339-53.

Access full text article on other devices

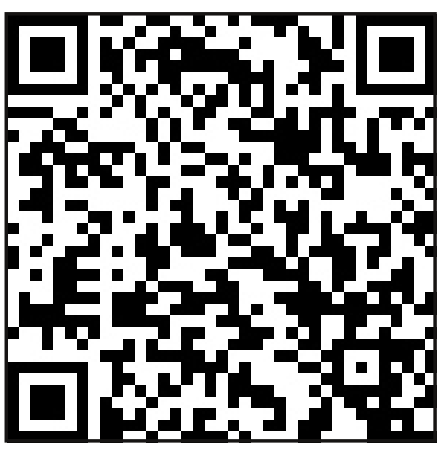

Access PDF of article on other devices

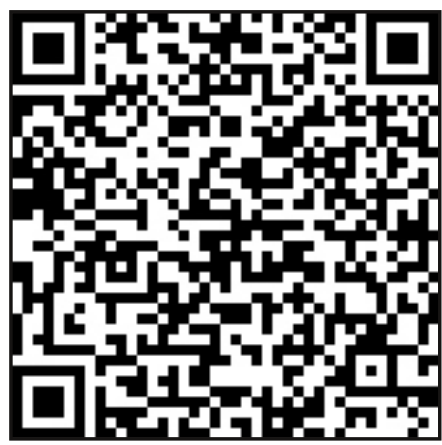

